Running title: DEX implant for retinal vein occlusion

\title{
Efficacy of Dexamethasone Intravitreal Implant For Refractory Macular Edema Caused by Retinal Vein Occlusion
}

Rehan M. Hussain, M.D., ${ }^{1}$ Thomas A. Ciulla, M.D., M.B.A ${ }^{1,2}$, Lauren M. Ciulla ${ }^{2}$, Bethany Sink ${ }^{2}$, Alon Harris, Ph.D. ${ }^{1}$

${ }^{1}$ Department of Ophthalmology, Indiana University School of Medicine, $1160 \mathrm{~W}$. Michigan St, Indianapolis, IN 46202

${ }^{2}$ Retina Service, Midwest Eye Institute, 200 W. $103^{\text {rd }}$ St, Indianapolis, IN 46290

Corresponding Author:

Thomas A. Ciulla, M.D., M.B.A.

Volunteer Clinical Professor of Ophthalmology

Indiana University School of Medicine

Retina Service, Midwest Eye Institute

200 W. $103^{\text {rd }}$ Street

Indianapolis, IN 46290

Thomasciulla@gmail.com

Phone: $317-817-1822$

Fax: $317-817-1898$

$\mathrm{TC}$ received contracted research funding from Acucela, Alcon, Ophthotec,

Thrombogenics, and Xoma within the past year. He currently has an employment

relationship with Ophthotech. AH would like to disclose that he receives remuneration

from CIPLA, Stemnion, Biolight, Nano Retina, AdOM, Science Based Health, Isarna

Therapeutics, and ONO Pharmaceuticals for serving as a consultant. AH also holds an

ownership interest AdOM, Oxymap and Nano Retina. All relationships listed above are pursuant to IU's policy on outside activities. None of the other authors have any financial disclosures. There are no conflicts of interest to report.

No funding was received for this work.

This is the author's manuscript of the article published in final edited form as:

Hussain, R., Ciulla, T., Ciulla, L., Sink, B., \& Harris, A. (2018). Efficacy of Dexamethasone Intravitreal Implant for Refractory Macular Edema Caused by Retinal Vein Occlusion. Retinal Cases \& Brief Reports, 12(4), 294-299. 
Key Words: BRVO, CRVO, corticosteroids, dexamethasone, macular edema, optical coherence tomography, Ozurdex

Summary Statement: Dexamethasone intravitreal implant effectively reduced recalcitrant macular edema caused by retinal vein occlusion. Visual acuity initially improved with treatment. However at one year follow-up, it worsened compared to baseline in both phakic and pseudophakic eyes. $50 \%$ of phakic eyes underwent cataract surgery and $23 \%$ of eyes developed ocular hypertension.

\section{$\underline{\text { Abstract }}$}

Purpose: To investigate efficacy of dexamethasone intravitreal implant (DEX) in treating refractory macular edema (ME) caused by retinal venous occlusion (RVO).

Methods: Retrospective chart review.

Results: 22 eyes with refractory ME caused by RVO were treated with a mean of 2.2 DEX over 12 months. Patient had previously received a mean of 7 treatments (laser, bevacizumab, and/or triamcinolone) for ME present for at least 4 months duration (mean 20.8 months $20.8 \pm 17.6$, range $4-72$ months) prior to starting DEX. Mean baseline visual acuity (VA) was 20/91 and mean central subfield thickness (CSFT) was $506 \mu \mathrm{m}$. DEX improved mean BCVA to 20/75 and 20/66 at 7 week and 6 month follow-up, although it worsened to 20/132 at 12 months. Mean CSFT improved to 292, 352, and $356 \mu \mathrm{m}$ at 7 week, 6 month, and 12 month follow-up respectively. There was a statistically significant association between number of DEX treatments and CSFT $\left(\mathrm{p}=3.28 \times 10^{-9}\right)$. There was a statistically significant association between number of days followed and BCVA $(\mathrm{p}=0.006) .6$ of $12(50 \%)$ phakic patients developed visually significant cataract requiring 
surgery. 5 of $22(23 \%)$ patients developed ocular hypertension (intraocular pressure $>30$ ), and consequently did not undergo further treatment with DEX.

Conclusions: DEX resulted in sustained anatomic reduction of RVO-associated

refractory ME, although this did not translate into long term BCVA improvement in either phakic or pseudophakic patients, possibly related to chronic structural alterations in the retina despite reduction of edema. 


\section{Introduction}

Vision loss from retinal vein occlusion (RVO) is frequently due to macular edema (ME). ${ }^{1}$ The pathogenesis of ME following RVO is related to a variety of factors, including hydrostatic effects from increased venous pressure, inflammatory cytokines, dysregulation of endothelial tight junctions, and increased amount of vascular permeability factors, including vascular endothelial growth factor (VEGF). ${ }^{2-4}$ Macular laser photocoagulation, intravitreal anti-VEGF agents, and intravitreal corticosteroids are commonly utilized treatments for macular edema caused by central retinal vein occlusion (CRVO) or branch retinal vein occlusion (BRVO). However, there are certain patients who develop refractory ME despite multiple treatments with the aforementioned modalities.

The dexamethasone $(0.7 \mathrm{mg}$ ) intravitreal implant (DEX implant; OZURDEX, Allergan, Inc., Irvine, CA) was approved in 2009 to treat macular edema caused by retinal vein occlusion. It is contained in a solid bioerodable polymer for sustained-release, and can exert clinical effects for three to six months. The OZURDEX GENEVA study showed that both the $0.35 \mathrm{~g}$ and $0.7 \mathrm{mg}$ DEX implant groups were both superior to sham in preventing visual acuity loss, and improving the rapidity and incidence of visual acuity

recovery in treatment-naïve eyes with ME secondary to CRVO or BRVO. ${ }^{5}$ The authors of this present study performed retrospective review of $0.7 \mathrm{mg}$ DEX implant used to treat RVO-associated macular edema that had been refractory to multiple prior treatments.

\section{Methods}


This retrospective, uncontrolled chart review studied patients diagnosed with refractory ME due to RVO, who were treated with their first DEX implant from March 2010 through July 2015. This project was reviewed by Indiana University's IRB and considered exempt. Fluorescein angiography was performed on each patient on initial presentation to the clinic. Only patients diagnosed with CRVO or BRVO were included.

Refractory ME was diagnosed if the patients experienced persistent ME of at least 4 months duration despite at least 2 prior treatments, including any combination of macular laser photocoagulation, intravitreal triamcinolone acetonide, intravitreal bevacizumab, or intravitreal ranibizumab. In those patients who had undergone macular laser treatment, a grid pattern had been applied to areas with diffuse leakage, between 500 and 3000 microns from the fovea; $532 \mathrm{~nm}$ laser was set to spot size of 50 microns at 0.05 to $0.1 \mathrm{sec}$.

Exclusion criteria included other causes of macular edema, such as diabetic retinopathy or neovascular age-related macular degeneration. Patients were excluded if the baseline best corrected visual acuity (BCVA) was better than 20/40, the central subfield thickness (CSFT) on spectral domain optical coherence tomography (OCT) was less than 300 microns, or if the foveal avascular zone (FAZ) was enlarged to greater than 1000 microns. A minimum of 6 months of follow-up was required to be eligible for the study.

Patient charts were reviewed for eligibility, and data were extracted regarding the patient's age, gender, previous interventions, and CSFT. The BCVA, CSFT, intraocular pressure (IOP), lens status, treatment dates from the initial visit and follow-up visits were recorded. Snellen visual acuity was converted to logarithm of minimal angle of 
resolution $(\log \mathrm{MAR})$ for statistical analysis. In cases of missing data points, the last observation was carried forward. Regression analysis was performed on the individual data to determine correlations between the independent variables of number of days follow-up or number of DEX implants and the dependent variables of logMAR VA or CSFT. Mean values for logMAR VA and CSFT, as well as standard deviations and range, were calculated at each follow-up visit.

On all visits, response to treatment was evaluated subjectively by Snellen visual acuity with best correction, and objectively by biomicroscopic examination and Zeiss Cirrus spectral domain OCT (Carl Zeiss Meditec, Jena, Germany). In all cases, the use of DEX implant and its potential risks and benefits were discussed with the patients before signing an informed consent. DEX implant was injected $3.5-4 \mathrm{~mm}$ posterior to the limbus under aseptic conditions. Subsequent injections were administered on an as needed basis for persistent macular edema on OCT affecting the foveal center.

\section{Results:}

The study included 22 eyes of 22 patients with ME caused by venous occlusion (10 patients with BRVO and 12 with CRVO). The mean age was $70 \pm 17.6$ years (range 46- 86 years). There were 9 males and 13 females. 12 patients were phakic (55\%) and 10 were pseudophakic (45\%) at the initiation of the study. All patients experienced ME for at least 4 months duration (mean $20.8 \pm 17.6$ months, range 4-72 months) prior to undergoing treatment with DEX implant (Table 1).

Prior to treatment with DEX implant, this group of eyes received an average of 7 prior treatments (22 eyes received 2-19 treatments including macular laser, intravitreal 
bevacizumab, ranibizumab, or triamcinolone acetonide). The mean washout period, in which no treatment was given prior to initial DEX implant, was $133 \pm 97$ days (median 117 days, range 38-402 days). See Table 2.

With respect to visual acuity, the mean BCVA prior to the initial DEX implant was $20 / 91(\operatorname{logMAR} 0.66 \pm 0.25)$. This improved to $20 / 75(\log M A R 0.57 \pm 0.32)$ at the first follow-up visit averaging 7 weeks later, and 20/66 (logMAR $0.52 \pm 0.25)$ at the 6month follow-up. By the 1 year follow-up, mean BCVA worsened to 20/131 (logMAR $0.82 \pm 0.42$ ), despite administration of a mean of $2.2 \pm 0.85 \mathrm{DEX}$ implants per patient (Table 3 and Figure 1). A statistically significant relationship was found between mean $\operatorname{logMAR}$ VA and number of days followed ( $\mathrm{p}=0.006)$, but not for mean logMAR VA and number of DEX implants administered $(p=0.99)$.

Compared to BRVO patients, who started with better mean BCVA, CRVO patients experienced a greater initial improvement of mean BCVA. Ultimately, BRVO patients maintained an improved 6-month and 1 year mean BCVA, while the CRVO patients experienced loss of mean BCVA (Table 3 and Figure 1).

With respect to macular edema as measured by OCT, prior to the initial DEX implant, CSFT for all patients averaged $506 \pm 150 \mu \mathrm{m}$. At the first follow-up visit at an average of 7 weeks later, there was meaningful improvement to a mean of $292 \pm 134 \mu \mathrm{m}$ (42\% reduction). Mean CSFT increased to $352 \pm 152 \mu \mathrm{m}$ and $356 \pm 131 \mu \mathrm{m}$ at 6 months (after mean $1.5 \pm 0.51$ DEX implants) and 12 months (after mean $2.2 \pm 0.85$ DEX implants) follow-up, respectively (Table 3 and Figure 2). A statistically significant relationship was found between mean CSFT and number of DEX implants administered $\left(\mathrm{p}=3.28 \times 10^{-9}\right)$, but not for mean CSFT and number of days followed $(\mathrm{p}=0.40)$. 
BRVO patients exhibited a more favorable baseline mean CSFT compared to the CRVO group. Mean CSFT improved meaningfully for both BRVO and CRVO groups after the first DEX implants, but worsened on subsequent visits (Table 3 and Figure 2). We performed analysis to determine whether duration of ME prior to treatment with DEX correlated with treatment response. Patients with ME duration $<12$ months had a greater response to the first treatment, as mean $\log \mathrm{MAR}$ improved by $0.23 \pm 0.07$ ( 0.60 to 0.37 ) and mean CSFT improved by $234 \pm 31 \mu \mathrm{m}(539 \mu \mathrm{m}$ to $305 \mu \mathrm{m})$. Patients with $>12$ months duration of ME had a smaller improvement, as mean logMAR improved by $0.08 \pm 0.07$ ( 0.55 to 0.47$)$ and mean CSFT improved by $199 \pm 52 \mu \mathrm{m},(479 \mu \mathrm{m}$ to $280 \mu \mathrm{m})$. The difference in $\log$ MAR VA was statistically significant $(\mathrm{p}=0.05)$, while the difference in CSFT was not significant $(\mathrm{p}=0.27)$.

With respect to intraocular pressure, mean IOP prior to the first DEX implant was $16.7 \pm 3.8$, which increased to $21.2 \pm 6$ at the first follow-up visit (Table 3). 5 of 22 patients $(22.7 \%)$ were discontinued from additional treatment with DEX implant, due to development of ocular hypertension (IOP $>30$ ). These patients had a minimum of 2 DEX implants (similar to the mean of 2.2 DEX implants for all patients) and hence were considered in the final analysis. All 5 patients showed improved IOP $(<21 \mathrm{~mm} \mathrm{Hg})$ after discontinuation of DEX implant and initiation of one topical IOP lowering agent.

Regarding lens status, 6 of 12 initially phakic patients (50\%) experienced cataract progression for which they underwent cataract surgery. This subset of patients was followed out to their most recent clinic visit (mean 970 days follow-up). Visually significant cataract was observed after a mean of $446 \pm 36$ days and $3.17 \pm 0.75$ DEX implants. The phakic patients showed mean baseline BCVA of 20/72 and one-year mean 
BCVA of 20/105. The baseline pseudophakic patients exhibited worse mean baseline BCVA of 20/119 and one-year BCVA of 20/170 (Figure 3). There were no cases of endophthalmitis, retinal detachment, or death throughout the study period.

\section{Conclusions}

This study evaluated outcomes of repeated DEX implant injections to treat refractory ME caused by RVO. The results suggest that DEX implant is effective in reducing CSFT for a sustained period, but improvements in BCVA were transient. The greatest improvement in mean CSFT (42\%) occurred after the first DEX implant,. The $\operatorname{logMAR}$ VA followed an undulating pattern, correlating with DEX treatment and its duration of action of approximately 3-4 months. The CRVO patients had a greater initial improvement in mean BCVA and mean CSFT, likely due to the fact that their baseline values were worse (with less potential for a ceiling effect), although ultimately only the BRVO subgroup maintained stable improvement in mean BCVA.

There was a high incidence of visually significant cataract (particularly posterior subcapsular cataracts) that developed in phakic patients receiving repeated DEX implants and this can partially account for the deteriorating BCVA. However, baseline pseudophakic patients had a similar deterioration of BCVA at one year follow-up, suggesting that cataract formation cannot fully account for deterioration in vision. Other studies have suggested that patients with chronic edema fare poorly. Even patients with macular edema for 6 months duration fare worse than those treated promptly; for example, in the pivotal Phase 3 randomized prospective clinical trial of ranibizumab for macular edema following CRVO (CRUISE), sham patients who crossed 
over at 6 months to ranibizumab treatment, did not recover to the same degree at 12 months, as those treated with ranibizumab from the start. ${ }^{6}$

In the current study, the neuroretinal atrophy caused by chronic macular edema may be responsible for the worsening of vision in these patients despite reduction of macular edema. It has been reported that patients with retinal vein occlusion with poor visual acuity $(\log M A R<1.0)$ despite resolution of macular edema had OCT findings of inner retinal thinning (suggesting atrophy). The patients with relatively good visual acuity $(\log$ MAR $<0.3)$ had the macular edema above the inner segments of the ellipsoid zone and had an intact ellipsoid zone more frequently after resolution of macular edema. ${ }^{7}$ Our analysis indicates that patients with duration of $\mathrm{ME}>12$ months prior to treatment with DEX had statistically significant less improvement in $\log$ MAR after one treatment compared to those with $<12$ months duration, which may support these findings, as greater duration of ME would be more likely to cause chronic structural alterations in the retina despite reduction of edema. The difference in reduction of macular thickness was not statistically significant between these two groups.

These results show a more notable improvement in CSFT but less improvement in VA compared to results reported by Sharareh et al, who studied eyes with RVO-related macular edema refractory to multiple bevacizumab injections. ${ }^{8}$ Alshahrani et al reported that a single DEX implant caused a statistically significant improvement in both VA and CSFT in patients with refractory ME, which peaked at one and three months, and then lost significance by 6 months. ${ }^{9}$ Our results followed a similar pattern of significant improvement of mean BCVA and mean CSFT within the first 7 weeks, with diminished effects by 3 month follow-up and onward, despite repeated DEX implants. 
Limitations of this study include its uncontrolled retrospective nature without a standardized refraction protocol, lack of standardized regimen prior to initiating DEX treatment, and limited sample size. Furthermore, statistical analyses in retrospective studies are inherently flawed with hindsight bias, and consequently meant to be exploratory in nature for hypothesis generation. Additionally, the washout period had a wide range of 38-402 days, which was likely due to poor follow up for selective patients that had failed to respond adequately to previous treatments. However consistent with prior studies, this study suggests that DEX may treat chronic RVO-related ME refractory to prior anti-VEGF treatment or laser photocoagulation, which is also consistent with the mechanism of action of corticosteroids. DEX implants reduce several pro-permeability proteins and inflammatory mediators, providing a multitargeted approach in treating RVO. ${ }^{10}$ Furthermore, in a recent large prospective trial of another corticosteroid, fluocinolone implant, for diabetic macular edema (DME), treatment was more effective in those eyes with chronic DME, and the authors speculated that chronic DME was driven by subclinical inflammation, compared to acute DME which is driven by VEGF. ${ }^{11}$ It is biologically plausible that a similar phenomenon occurs in chronic refractory ME due to RVO. Nevertheless, this current study, along with the prior studies discussed herein, suggest that chronic edema is best avoided, given the guarded prognosis. Clearly, further study of treatment regimens for refractory and/or chronic ME is warranted, given the visual disability caused. 


\section{References}

1. Karia N. Retinal vein occlusion: Pathophysiology and treatment options. Clin Ophthalmol 2010;4:809-16.

2. Rehak J, Rehak M. Branch retinal vein occlusion: Pathogenesis, visual prognosis, and treatment modalities. Curr Eye Res 2008;33:111-31.

3. Antonetti DA, Barber AJ, Khin S, et al. Vascular permeability in experimental diabetes is associated with reduced endothelial occludin content: Vascular endothelial growth factor decreases occludin in retinal endothelial cells. Penn state retina research group. Diabetes 1998;47:1953-9.

4. Campochiaro PA, Hafiz G, Shah SM, et al. Ranibizumab for macular edema due to retinal vein occlusions: Implication of vegf as a critical stimulator. Mol Ther 2008;16:791-9.

5. Haller JA, Bandello F, Belfort R, Jr., et al. Randomized, sham-controlled trial of dexamethasone intravitreal implant in patients with macular edema due to retinal vein occlusion. Ophthalmology 2010;117:1134-46 e3.

6. Campochiaro PA, Brown DM, Awh CC. Sustained benefit from ranibizumab for macular edema following central retinal vein occlusion: twelve-month outcomes of a phase III study. Ophthalmology 2011;118(10):2041-9.

7. Schroder K, Ackermann P, Brachert L, et al. [Does OCT morophology provide indications for prognosis of visual acuity after vein occlusion?: SD-OCT analysis in retinal vein occlusion before and after resolution of initial macular edema]. Ophthalmologe 2016;113(6):500-6

8. Sharareh B, Gallemore R, Taban M, et al. Recalcitrant macular edema after intravitreal bevacizumab is responsive to an intravitreal dexamethasone implant in retinal vein occlusion. Retina 2013;33:1227-31.

9. Alshahrani ST, Dolz-Marco R, Gallego-Pinazo R, et al. Intravitreal dexamethasone implant for the treatment of refractory macular edema in retinal vascular diseases: Results of the kkesh international collaborative retina study group. Retina 2015;36(1):131-6.

10. Campochiaro PA, Hafiz G, Mir TA, et al. Pro-permeability factors after dexamethasone implant in retinal vein occlusion; the ozurdex for retinal vein occlusion (orvo) study. Am J Ophthalmol 2015;160(2):313-321.

11. Cunha-Vaz J, Ashton P, Iezzi R, et al. Sustained delivery fluocinolone acetonide vitreous implants: Long-term benefit in patients with chronic diabetic macular edema. Ophthalmology 2014;121:1892-903. 


\section{Figure Captions:}

Figure 1. Mean $\log$ MAR visual acuity in relation to number of days of follow-up after first treatment in eyes treated with $0.7 \mathrm{mg}$ intravitreal dexamethasone implant. Visual acuity followed a saw-toothed pattern that roughly correlated to the injection schedule, although at last follow-up it had significantly worsened compared to baseline. The BRVO group had relatively favorable outcomes compared to the CRVO group.

Figure 2. Mean central subfield thickness (CSFT) in relation to number of days of follow-up after first treatment in eyes treated with $0.7 \mathrm{mg}$ intravitreal dexamethasone implant. CSFT reduced drastically in the first 7 weeks, although it later plateaued on subsequent visits.

Figure 3. Subgroup analysis of baseline phakic vs. pseudophakic eyes indicates that both groups had initial improvement of mean $\log$ MAR visual acuity with intravitreal dexamethasone treatment. However, after approximately 180 days follow-up, visual acuity began to worsen in both groups. 


\section{Table 1. Baseline patient characteristics}

\begin{tabular}{|l|l|}
\hline Gender & $9 / 22$ male, $11 / 22$ female \\
\hline Mean age at diagnosis & $70 \pm 17.6$ years (range 46-86 years) \\
\hline Central Retinal Vein Occlusion & $12 / 22$ patients \\
\hline Branch Retinal Vein Occlusion & $10 / 22$ patients \\
\hline Mean duration of macular edema & $20.8 \pm 17.6$ months (range $4-72$ months) \\
\hline Mean visual acuity & $20 / 91($ logMAR $0.66 \pm 0.25)$ \\
\hline Mean central subfield thickness & $506 \pm 150 \mu \mathrm{m}$ (range 304-871 $\mu \mathrm{m})$ \\
\hline Mean intraocular pressure & $16.7 \pm 3.8$ mmHg (range 12-23) \\
\hline Lens status & $12 / 22$ phakic, $10 / 22$ pseudophakic \\
\hline
\end{tabular}

$\log \mathrm{MAR}=\operatorname{logarithm}$ of the minimum angle of resolution 
Table 2. Summary of mean number of treatments given for macular edema prior 0.7 mg DEX implant.

$\begin{array}{lllllll} & \text { \#Laser } & \text { \#Tri } & \text { \#Bev } & \text { \#Ran } & \text { \#Bev-Tri } & \text { Total } \\ \text { Mean } & 0.55 & 0.14 & 5.59 & 0.14 & 0.73 & 7 \\ \text { SD } & 0.86 & 0.47 & 4.69 & 0.64 & 1.32 & 4.83 \\ \text { Minimum } & 0 & 0 & 0 & 0 & 0 & 2 \\ \text { Maximum } & 3 & 2 & 17 & 3 & 3 & 19 \\ \text { \# Eyes } & 8 & 2 & 20 & 1 & 7 & \\ \text { treated } & & & & & & \\ \text { Final } & 5 / 22 \text { eyes } & 0 / 22 \text { eyes } & 14 / 22 \text { eyes } & 0 / 22 \text { eyes } & 3 / 22 \text { eyes } & \\ \text { treatment* } & & & & & & \\ \text { Mean } & 135 \text { days } & \text { N/A } & 144 \text { days } & \text { N/A } & & \\ \text { washout } & & & & & & \end{array}$

Tri $=$ intravitreal triamcinolone acetonide; $\mathrm{Bev}=$ intravitreal bevacizumab; $\mathrm{Ran}=$ intravitreal ranibizumab; Bev-Tri = combination of bevacizumab and triamcinolone; SD $=$ standard deviation; N/A = not applicable; * = prior to starting DEX implant 
Table 3. Mean values of logMAR Visual Acuity, CSFT and IOP data in relation to number of days of treatment and number DEX implants.

\begin{tabular}{|c|c|c|c|c|}
\hline \multicolumn{5}{|l|}{$\begin{array}{r}\text { Total } \\
\text { patients }\end{array}$} \\
\hline Day & \# DEX & $\log M A R$ & $\operatorname{CSFT}(\mu \mathrm{m})$ & IOP (mmHg) \\
\hline 0.0 & 0.0 & $0.66 \pm 0.25$ & $506 \pm 150$ & $16.7 \pm 3.8$ \\
\hline $52.1 \pm 25.6$ & $1.0 \pm 0$ & $0.57 \pm 0.32$ & $292 \pm 134$ & $21.0 \pm 6.0$ \\
\hline $110.7 \pm 32.3$ & $1.1 \pm 0.2$ & $0.70 \pm 0.37$ & $397 \pm 176$ & $17.1 \pm 3.5$ \\
\hline $179.5 \pm 40.1$ & $1.5 \pm 0.5$ & $0.52 \pm 0.25$ & $352 \pm 152$ & $19.1 \pm 5.9$ \\
\hline $239.6 \pm 52.0$ & $1.8 \pm 0.6$ & $0.68 \pm 0.34$ & $348 \pm 121$ & $17.8 \pm 5.4$ \\
\hline $294.0 \pm 79.6$ & $2.0 \pm 0.7$ & $0.43 \pm 0.29$ & $349 \pm 152$ & $17.9 \pm 6.9$ \\
\hline $352.6 \pm 142.2$ & $2.2 \pm 0.9$ & $0.82 \pm 0.42$ & $356 \pm 131$ & $16.0 \pm 4.5$ \\
\hline \multicolumn{5}{|l|}{ CRVO only } \\
\hline Day & \# DEX & $\log M A R$ & CSFT $(\mu \mathrm{m})$ & IOP (mmHg) \\
\hline 0.0 & 0.0 & $0.76 \pm 0.30$ & $564 \pm 161$ & $16.9 \pm 4.6$ \\
\hline $52.8 \pm 34.3$ & $1.0 \pm 0$ & $0.62 \pm 0.36$ & $312 \pm 168$ & $21.6 \pm 6.9$ \\
\hline $111.3 \pm 43.1$ & $1.1 \pm 0.3$ & $0.83 \pm 0.46$ & $429 \pm 203$ & $17.3 \pm 4.1$ \\
\hline $182.6 \pm 48.3$ & $1.6 \pm 0.5$ & $0.58 \pm 0.32$ & $375 \pm 191$ & $20.2 \pm 7.6$ \\
\hline $246.9 \pm 65.7$ & $1.9 \pm 0.7$ & $0.76 \pm 0.40$ & $370 \pm 142$ & $18.1 \pm 5.7$ \\
\hline $316.3 \pm 89.8$ & $2.3 \pm 0.5$ & $0.66 \pm 0.35$ & $382 \pm 194$ & $19.8 \pm 8.6$ \\
\hline $399.5 \pm 157.8$ & $2.5 \pm 0.7$ & $0.98 \pm 0.49$ & $401 \pm 152$ & $16.4 \pm 5.6$ \\
\hline \multicolumn{5}{|l|}{ BRVO only } \\
\hline Day & \# DEX & $\log M A R$ & $\operatorname{CSFT}(\mu \mathrm{m})$ & IOP (mmHg) \\
\hline 0.0 & 0.0 & $0.51 \pm 0.14$ & $436 \pm 103$ & $16.4 \pm 2.7$ \\
\hline $51.2 \pm 9.5$ & $1.0 \pm 0$ & $0.51 \pm 0.28$ & $267 \pm 75$ & $20.2 \pm 4.9$ \\
\hline $109.9 \pm 12.4$ & $1.0 \pm 0$ & $0.47 \pm 0.21$ & $359 \pm 137$ & $17.0 \pm 3.0$ \\
\hline $175.7 \pm 29.3$ & $1.4 \pm 0.5$ & $0.43 \pm 0.14$ & $324 \pm 86$ & $17.8 \pm 2.7$ \\
\hline $230.7 \pm 28.0$ & $1.7 \pm 0.5$ & $0.54 \pm 0.27$ & $321 \pm 89$ & $17.4 \pm 5.4$ \\
\hline $267.2 \pm 58.8$ & $1.8 \pm 0.8$ & $0.39 \pm 0.19$ & $309 \pm 67$ & $15.6 \pm 3.3$ \\
\hline $296.4 \pm 101.4$ & $1.9 \pm 0.9$ & $0.47 \pm 0.27$ & $302 \pm 75$ & $15.4 \pm 2.8$ \\
\hline $319.5 \pm 126.4$ & $1.9 \pm 0.9$ & $0.40 \pm 0.20$ & $358 \pm 118$ & $15.3 \pm 2.9$ \\
\hline
\end{tabular}

$\operatorname{LogMAR}=$ logarithm of the minimum angle of resolution; CSFT $=$ central subfield thickness; IOP = intraocular pressure; $\mathrm{mmHg}=$ millimeters of mercury; DEX = Dexamethasone intravitreal implant; BRVO = Branch Retinal Vein Occlusion; CRVO = Central Retinal Vein Occlusion; 


\section{Mean Visual Acuity (logMAR) vs. Number of Days Followed}
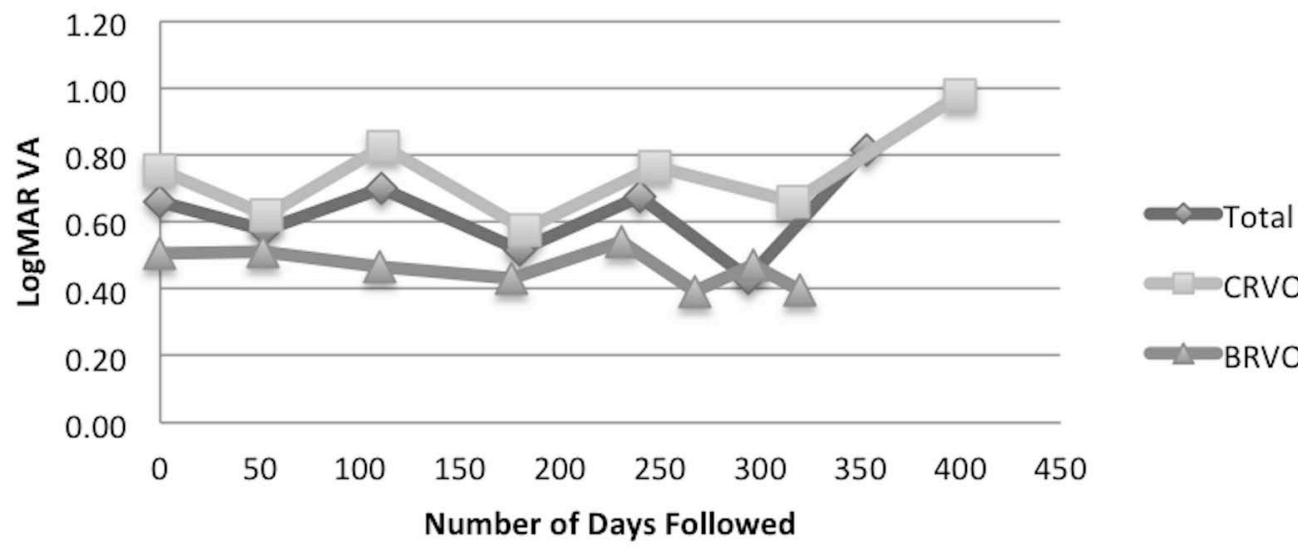


\section{Mean Central Subfield Thickness (CSFT) vs. Number of Days Followed}

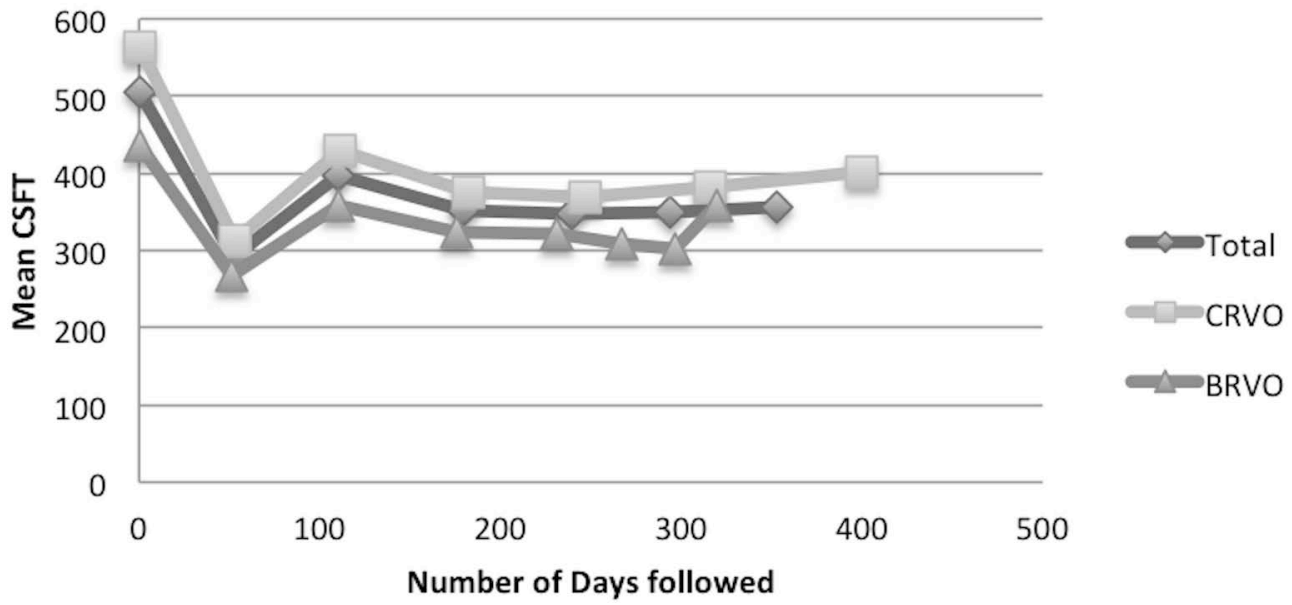




\section{Mean Visual Acuity (logMAR) vs. Number of Days Followed}

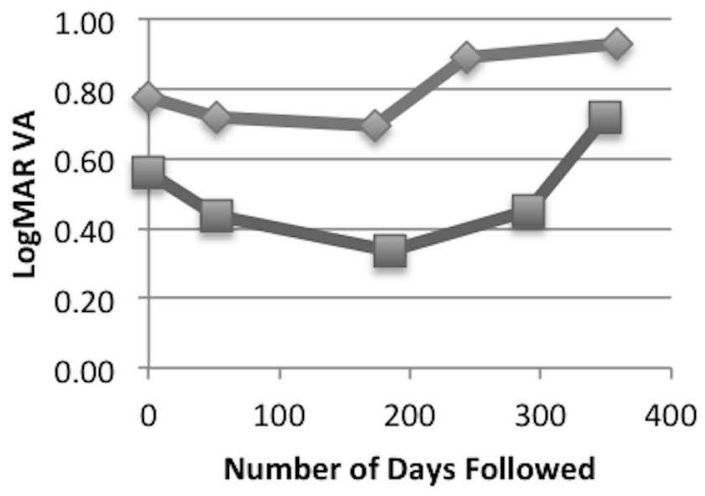

$\approx$ Baseline pseudophakic

- Baseline phakic

Number of Days Followed 